\title{
Electrochemical Functionalisation of Nanotubes Films: Growth of Aryl Chains on Single-Walled Carbon Nanotubes
}

\author{
Pierre R. Marcoux, ${ }^{* a}$ Philippe Hapiot, ${ }^{b}$ Patrick Batail, ${ }^{a}$ and Jean Pinson ${ }^{c}$ \\ ${ }^{a}$ Chimie Inorganique, Matériaux aux Interfaces (CIMI), FRE 2447, CNRS-Université d'Angers, bât. K, 2 \\ boulevard Lavoisier, 49045 Angers, France. Fax: +44 (0)21 6934470 ; Tel: +44 (0)21 6394297 ; E-mail: \\ prmarcoux@yahoo.fr \\ ${ }^{b}$ Synthèse et Electrosynthèse Organiques (SESO), UMR 6510, CNRS-Université de Rennes 1, Campus de \\ Beaulieu, bât. 10C, 35042 Rennes Cedex, France. Fax: +33 (0)2 23235939 ; Tel: +33 (0)2 23236732 ; E-mail: \\ philippe.hapiot@univ-rennes1.fr \\ ${ }^{c}$ Laboratoire d'Electrochimie Moléculaire, UMR 7591, CNRS-Université Paris 7, 2 place Jussieu, Case 7107, \\ 75251 Paris Cedex 05, France. Fax: +33 (0)1 44277625 ; Tel: +33 (0)1 44272801 ; E-mail: \\ jean.pinson@laposte.net
}

\section{This submission was created using the RSC Article Template (DO NOT DELETE THIS TEXT) (LINE INCLUDED FOR SPACING ONLY - DO NOT DELETE THIS TEXT)}

Covalent exohedral derivatizations of HipCo nanotubes, through electrochemical reduction of aryldiazonium salts, is described. Four different aryldiazonium salts have been used under the same experimental conditions: 4-bromophenyl, 4-chloromethylphenyl, 4sulfophenyl and 4-carboxyphenyl. Derivatized samples were characterized through X-Ray Photoelectron Spectroscopy and MicroRaman diffusion. The evolution of the spectrum (area of the D-band), as a function of the number of grafted groups, led us to the conclusion that the electrochemical reduction of aryldiazonium into radicals give rise to the growth of aryls chains on the sidewalls of nanotubes.

\section{Introduction}

With novel structural, electronic and mechanical properties, 1,2 single-walled carbon nanotubes (SWNTs) constitute an important new form of carbon that could find applications in many fields, ${ }^{3}$ depending on the possibilities to perform chemical modifications on the SWNTs walls. For example, the grafting of appropriate moieties on nanotubes may provide a chemical selectivity for nanotube-based SPM probes, ${ }^{4}$ improve solubility and ease of dispersions, ${ }^{5,6}$ supply a specificity for SWNTs-based molecular sensors, ${ }^{7}$ facilitate self-assembling onto surfaces, ${ }^{8,9}$ or provide a chemical attachment to polymer matrices in composites. ${ }^{10}$ According to Hirsch, ${ }^{11}$ the different approaches to the functionalisation of SWNTs may be classified into five categories: a) defect-group functionalisation, ${ }^{12,13}$ b) covalent sidewall functionalisation, 14,15 c) noncovalent exohedral functionalisation with amphiphilic molecules ${ }^{16}$ or surfactant, ${ }^{17}$ d) noncovalent exohedral functionalisation with polymers, ${ }^{18}$ and e) noncovalent endohedral functionalisation with, for example, $\mathrm{C}_{60} .{ }^{19}$ The grafting procedure considered in this article deals with the classified covalent sidewall functionalisations. It is based on the electrochemical reduction of an aryldiazonium salt. Reduction of these salts gives highly reactive aryl radicals that covalently attach to the nanotube surface. This methodology, discovered at the beginning of the nineties, ${ }^{20}$ has been first applied to carbon surfaces such as glassy carbon, ${ }^{21}$ HOPG graphite, ${ }^{22}$ carbon fibers, ${ }^{23}$ carbon felts ${ }^{24}$ or carbon black. ${ }^{25}$ It was recently applied to SWNTs, either on buckypapers, ${ }^{26}$ or on individual small bundles. ${ }^{27}$ It constitutes a simple, flexible and promising functionalisation method since it is specific (unlike methods based on strong oxidizing conditions, such as acidic treatments) and provides a one-step procedure for the grafting of a widespread variety of moieties. A large number of various aryldiazonium salts are indeed readily available, or can be prepared from the corresponding amine.

Our approach focuses on the electrochemical functionalisation of single-walled carbon nanotubes, using a buckypaper as an electrode. A special attention has been focused to the possibility of producing multilayer modifications through an apparent polymerisation process. Three types of diazonium salts bearing different substituents on the aryl groups were used: 4bromophenyl, 4-chloromethylphenyl and 4-carboxyphenyl diazonium. Then, increasing amounts of sidewall derivatization can be correlated to a consistent evolution of the Raman spectra of the grafted nanotubes. In addition, the comparison between the spectroscopic data coming from X-Ray Photoelectron Spectroscopy and Raman diffusion allow us to investigate the growth of aryl chains on SWNTs.
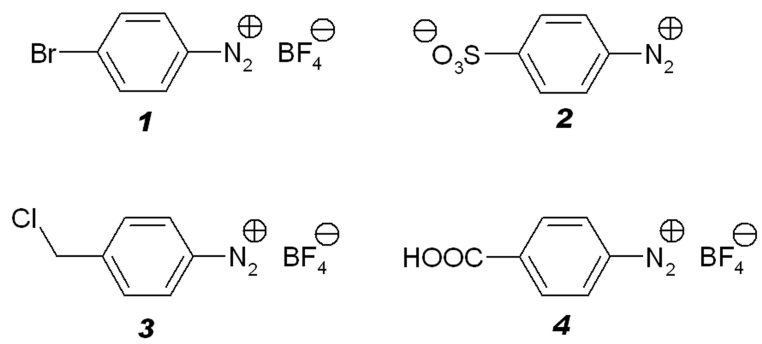

Fig. 1 Aryldiazonium salts used in this work to derivatize single-wall carbon nanotubes.

\section{Experimental Section}

Reagents. As-prepared SWNTs were obtained from Carbon Nanotechnologies Incorporated (Houston TX, http://www.cnanotech.com). The method by which these nanotubes were prepared is called HiPco process and is based on the catalytic decomposition of carbon monoxide. ${ }^{28}$ They were not purified before functionalisation, in order to avoid any oxidation of the tubes before grafting. The dominant impurity is the metal catalyst, which is encased in thin carbon shells (2-3 graphitic layers) and distributed throughout the sample as $2-5 \mathrm{~nm}$ diameter particles. We performed mass elemental analysis on asprepared SWNTs and the following molar ratios were obtained: $\mathrm{C} / \mathrm{Fe}=20,8$ and $\mathrm{O} / \mathrm{Fe}=2,1$ (iron catalyst is partially oxidized). Futhermore, we observed very small amounts of amorphous carbon. It was sometimes adsorbed on bundles, and most often on catalytic particles. Concerning aryldiazonium salts, the Figure 
1 shows the four experimented compounds. Compound 1 was obtained from Aldrich and compound 2 from Fluka. Other diazonium salts, chloromethylphenyl diazonium tetrafluoroborate $\mathbf{2}$ and 4-carboxyphenyl diazonium tetrafluoroborate 4 , were prepared by standard diazonation of the corresponding amines with $\mathrm{NaNO}_{2}$ in acidic medium. ${ }^{29}$

Electrode Preparation and Electrochemical Grafting. In order to handle easily large surfaces of nanotube thin films, SWNTs were deposited on a non adherent support. We chose as a support a filtration membrane made of Teflon (Sartorius, $1 \mu \mathrm{m}$ $\varnothing)$ since it is porous, non-adhesive, chemically inert and electrically insulating. SWNTs are first sonicated in ethanol (spectrosol quality) for 10 minutes. The obtained dispersion is then immediately filtered through a $1 \mu \mathrm{m}$ Teflon membrane, so as to deposit a thin layer of SWNTs $(\sim 2 \mu \mathrm{m}$, measured by scanning electronic microscopy) on the support. After drying, a rectangle is cut in the membrane and four holes are made at the corners. A copper wire runs through these holes, in order to form a rigid frame. Electric contact is ensured by a silver paint point (PROVAC AG, silver print) between the copper frame and the nanotubes layer. The prepared nanotubes film is used as the working electrode in a three-electrode cell and immersed in an acetonitrile solution containing the aryldiazonium salt $\left(10^{-2} \mathrm{M}\right)$ and the supporting electrolyte $\left(10^{-1} \mathrm{M}\right)$. Care is taken not to immerse the upper part of the nanotube film supporting the silver paint point. The counter-electrode is made with a graphite crucible that serves as a container for the acetonitrile solution at room temperature. The diazonium salt is reduced by applying a potential of $-1 \mathrm{~V} / \mathrm{SCE}$ and applied for a period of $30 \mathrm{~min}$. Oxygen is removed from the solution by bubbling argon during the experiment. The portion of the membrane that is not immersed in the solution is excised after reaction and the remainder is soaked in hot acetonitrile for $30 \mathrm{~min}$ to remove the aryldiazonium that has not reacted with the nanotubes films. It is then low density sonicated at room temperature for $5 \mathrm{~min}$ in acetonitrile, then in chloroform, acetone and ether. During these washing, a slight fraction of SWNTs leaves the film and can be recovered through filtration. The washed functionalised nanotubes are finally dried under air at $80^{\circ} \mathrm{C}(24 \mathrm{~h})$ and under primary vacuum at room temperature $(48 \mathrm{~h})$. We noticed that none of the functionalised samples were soluble in the solvents used for washing, also in dimethylformamide and 1,2-

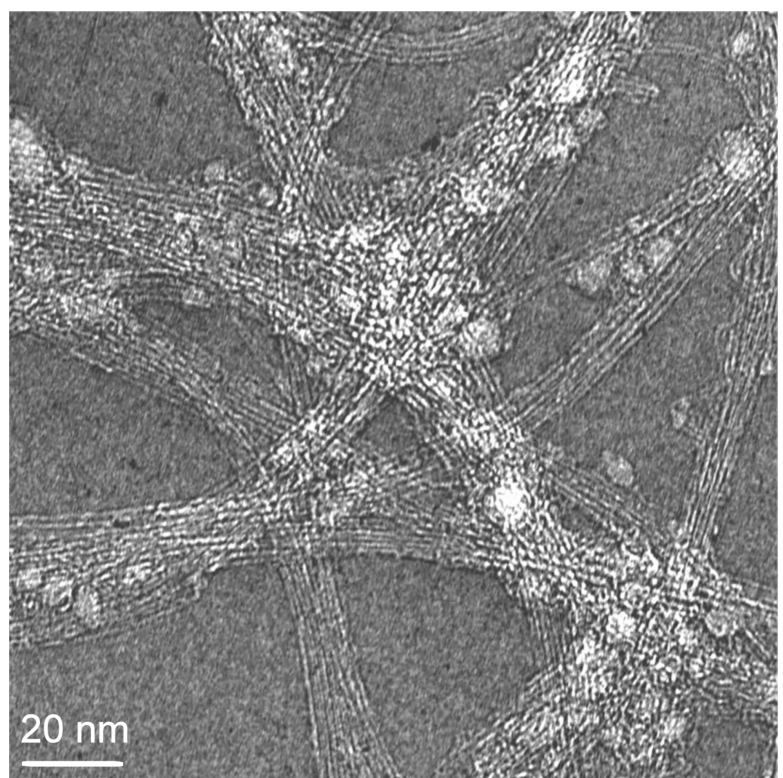

Fig. 2 High-resolution transmission electron microscopy image of a film of nanotubes, electrochemically functionalised with 4-bromophenyl diazonium. dichlorobenzene.

Spectroscopic Measurements. Raman data were recorded on a Jobin Yvon T64000 spectrometer (CNRS-Université de Nantes), equipped with a cooled charged coupled-device (CCD) detector. The scattering signal was collected through a microscope in a backscattering configuration. All spectra were obtained at room temperature, with a resolution of $2 \mathrm{~cm}^{-1}$. The laser power on the sample was limited to $2 \mu \mathrm{W}$ to avoid heating of the samples.

The XPS analyses were performed with a Leybold (LHS 12) spectrometer (CNRS-Université de Nantes). The ionising radiation $\left(\mathrm{K} \alpha_{1} \alpha_{2}\right.$ of $\left.\mathrm{Mg}\right)$ was provided by a nonmonochromatized X-Ray source, working at an acceleration tension of $12 \mathrm{kV}$ and an emission current of $10 \mathrm{~mA}$. Argon etching was not performed on the samples, in order to avoid any damage of the grafted groups.

Structural Characterization. SWNTs were observed, before and after functionalisation, with a Hitachi H9000-NAR $(300 \mathrm{kV})$ transmission electron microscope (TEM). Samples were
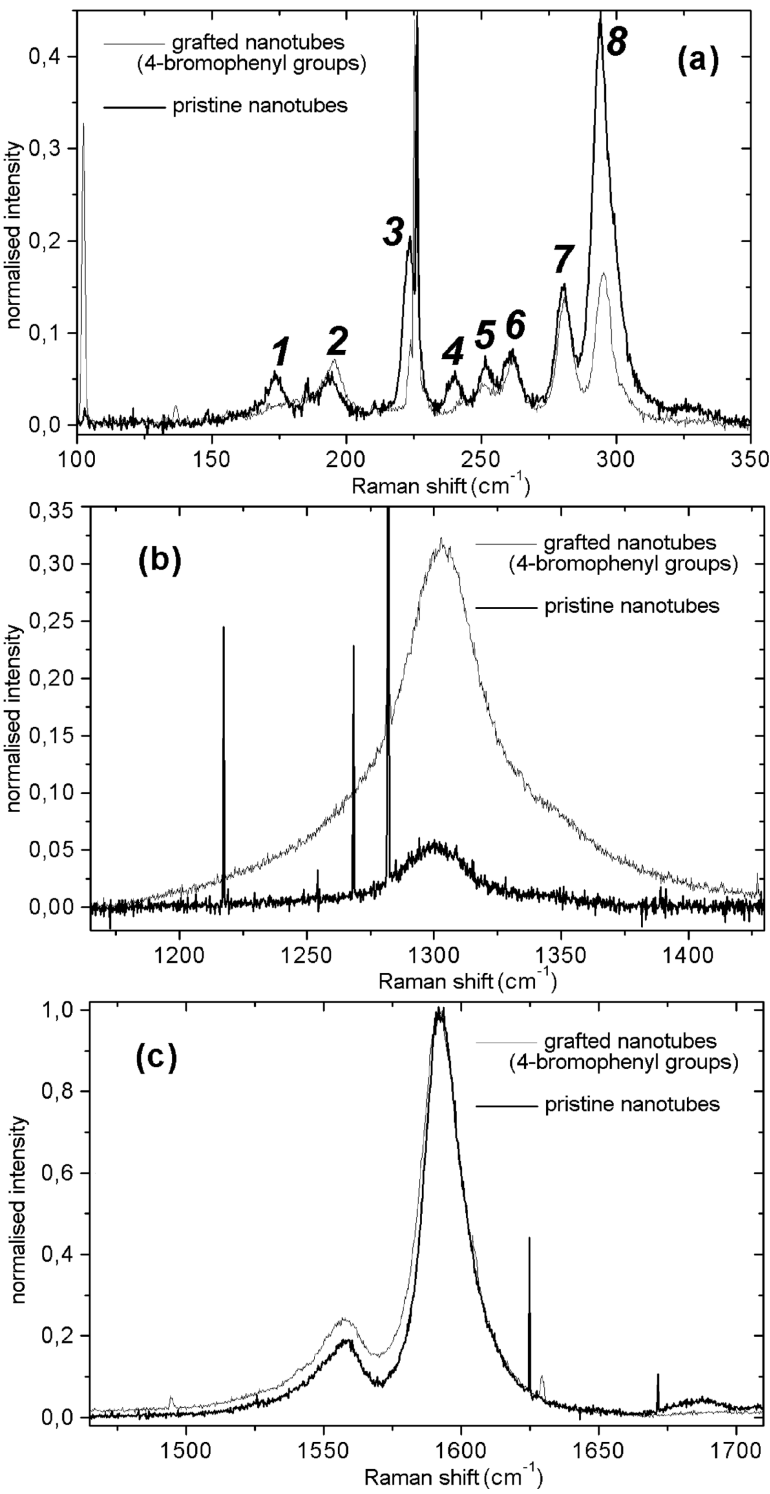

Fig. 3 Micro-Raman diffusion data collected at $676 \mathrm{~nm}$. Intensities are normalised in such a way that the intensity of the strongest tangential modes is 1 . The Figures $\mathrm{a}, \mathrm{b}$ and $\mathrm{c}$ plot the normalised intensities of, respectively, radial modes, D-band and tangential modes. 
prepared by evaporating an alcoholic solution of the nanotubes onto an amorphous carbon membrane.

\section{Results and discussion}

As a first example of grafting through the reduction of an aryldiazonium salt, we chose to modify the SWNTs by 4bromophenyl groups (1) since bromine atoms give a strong signal in XPS, allowing for an easy detection of the bonded moieties, even at low derivatization ratio. This diazonium has already been used before, for the derivatisation of the same kind of SWNTs (HipCo tubes), and these experiments allow a comparison with previously published results. ${ }^{26} 4-$ chloromethylphenyl (3) and 4-carboxyphenyl (4) groups were also considered in the purpose of performing later chemical reaction couplings with the attached groups, either through a nucleophilic substitution of chlorine, or through an esterification reaction of the carboxylic acid. Finally, the grafting of the sulfonate compound $\mathbf{2}$ was considered as a way for providing ionic functionalised nanotubes. Due to repulsive coulombic interactions, ionic grafted SWNTs are expected to be more soluble than neutral ones.

After electrochemical modification, the SWNTs were first examined by transmission electron microscopy. Structural characterization by the help of this technique showed that for all the considered aryldiazonium, the tubular structure is clearly maintained after the functionalisation (Figure 2). Thermogravimetric analyses were also performed on pristine sample, and on nanotubes grafted with 4-bromophenyl groups (nanotubes were previously scratched and taken off from the Teflon membrane). The conditions for both these experiments were : $5^{\circ} \mathrm{C} / \mathrm{min}$, under air, from $20^{\circ} \mathrm{C}$ to $850^{\circ} \mathrm{C}$. The thermogram of gratfed nanotubes showed only one step $\left(510^{\circ} \mathrm{C}-550^{\circ} \mathrm{C}\right)$ in the falling off of the mass. So it was not possible to highlight a distinct loss of mass specific to the grafted groups. Some thermal treatment was also tried under agon, at $500^{\circ} \mathrm{C}$, in order to
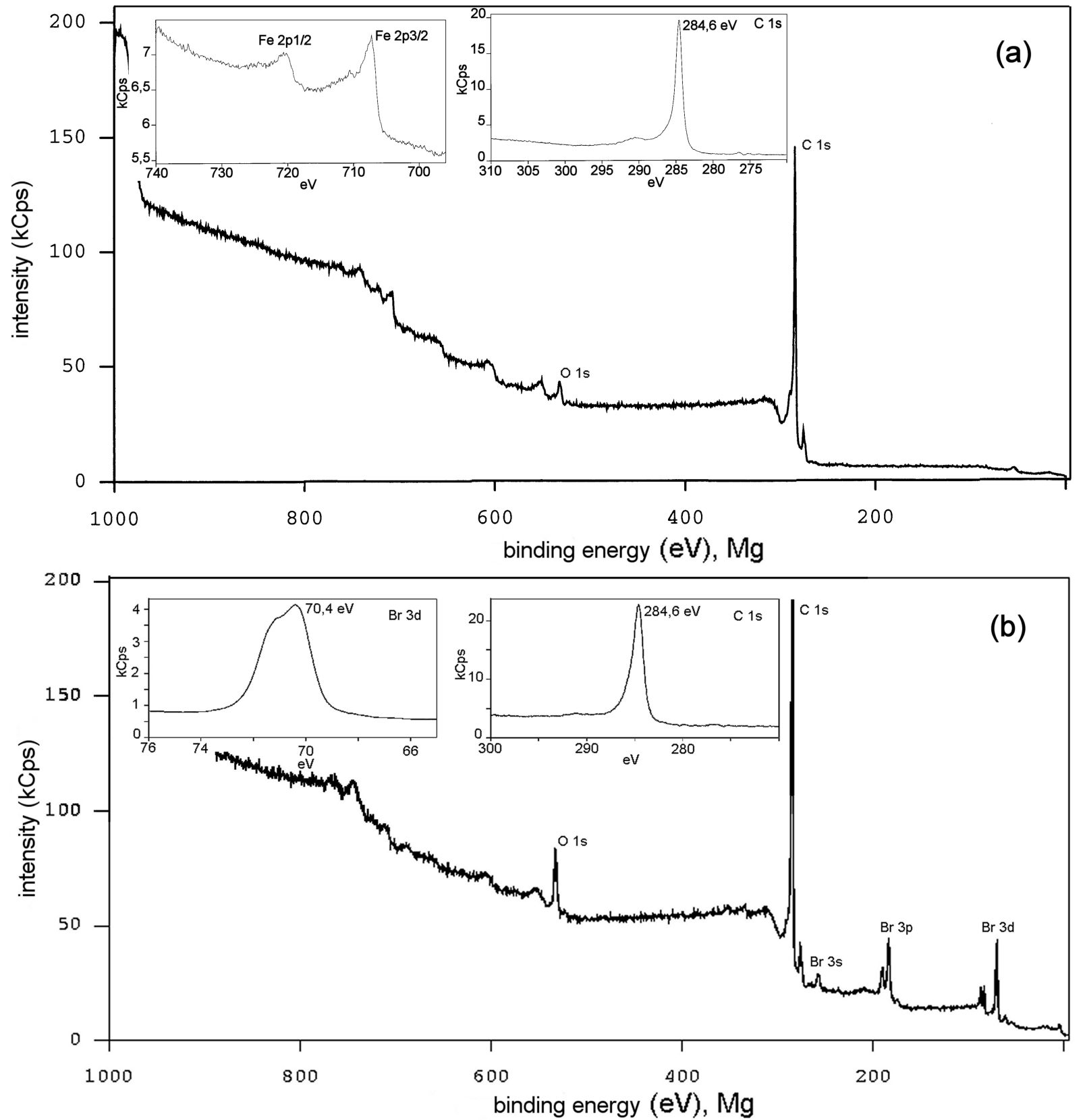

Fig. 4 (a) XPS spectrum of a film of pristine nanotubes (b) XPS spectrum of a film of nanotubes, electrochemically functionalised with 4-bromophenyl diazonium (aryldiazonium salt: $10^{-2} \mathrm{M}$; electrolyte: $\left(\mathrm{C}_{4} \mathrm{H}_{9}\right)_{4} \mathrm{NPF}_{6} 10^{-2} \mathrm{M} ;-1 \mathrm{~V} / \mathrm{SCE}$ applied for 30 minutes). The $\mathrm{O} 1 \mathrm{~s}$ peak is due essentially (i) to the oxidised iron catalytic nanoparticles, (ii) to carbons of the sidewalls that are oxidised during the purification of the HipCo tubes. 
recover the pristine nanotubes and to measure the mass of grafted groups. After such a treatment we realised, through TEM observation and Raman spectroscopy, that most of the nanotubes were destroyed (loss of the breating radial modes and very broadened tangential modes).

To go in deeper investigations concerning the spectroscopic characterization of the functionalised nanotubes, Micro-Raman spectroscopy appears as an efficient method to outline the modifications of the structure of the sidewalls due to the covalent grafting. As we have previously shown in the case of the fluorination of SWNTs, ${ }^{30}$ an increasing amount of sidewall derivatization is consistent with: (i) a decrease of the relative intensities of the radial modes, without any change of the frequencies; (ii) an increase of the relative intensity of the Dband, without any frequency shift; (iii) a strong broadening of the tangential modes, with a downshift frequency. Similar evolutions are expected after the electrochemical modification due to the binding of the aryl groups on the SWNTs walls. This is indeed what we observed for the radial modes and the D-band, as illustrated in the graphs (a) and (b) of the Figure 3, showing the spectrum of a sample electrochemically grafted with 4bromophenyl diazonium tetrafluoroborate. In contrast, tangential modes are hardly modified after aryl grafting (Figure $3 \mathrm{c}$ ): only a slight broadening can be seen and there is no frequency shift. The evolutions we observed concerning the relative intensities of radial modes and D-band are the same as those previously reported by Bahr et al. ${ }^{26}$ We have tried to see some "kinetics" of Raman D-band growth (growth of the relative area of D-band, as a function of time during which potential is applied). We have not managed yet to observe a clear trend because of difficulties in controlling local current densities. In our actual cell, it is indeed difficult to put every time the work electrode in the same position, compared to the two other electrodes. Current research is directed towards improving our electrochemical cell.

In complement of Raman diffusion experiments, X-Ray Photoelectron Spectroscopy allows the determination of the stoichiometry $\mathrm{C}_{\mathrm{n}} \mathrm{R}(\mathrm{R}=$ aryl group) i.e. the number of bonded aryl groups versus the number of carbons of the nanotube, by monitoring the signals specific of the elements attached to the grafted groups (bromine for 1, see Figure 1, sulfur for 2, chlorine for 3 and oxygen for 4). A noticeable difference between MicroRaman experiment and XPS lies in the size of the probed region. This question becomes important when the homogeneity of a functionalised nanotubes film is investigated. The probed zone with Raman diffusion is very deep, more than $10 \mu \mathrm{m}$ (that is, more than the whole thickness of the nanotube film, about 2 $\mu \mathrm{m}$ ), but is not widespread in the plane of the film (about $1 \mu \mathrm{m}$ radius). Raman spectroscopy can therefore be used as a way for checking the horizontal homogeneity, in the plane of the film. In contrast, the zone probed through XPS is widespread horizontally (several $\mathrm{mm}^{2}$ ), but with very little penetration depth (around $6 \mathrm{~nm}) .^{31,32}$ Since this sampling depth is several times larger than the diameter of a HiPco SWNT $(c a .0 .7 \mathrm{~nm}),{ }^{33}$ we can measure the variations of the chemical composition of SWNTs. The XPS technique can be used to test the homogeneity of the film as a function of its depth. In our case, horizontal homogeneity was investigated through Raman diffusion: all the samples, regardless of the aryl group, display the same signal intensity in all area of the sample indicating that all point of the surface has equally reacted with the electrogenerated radicals. For vertical homogeneity, we can only perform XPS measurements on the upper side of the film (the one that was facing the solution during the electrochemical process) and on the lower side (the one that was facing the Teflon membrane), which led approximately to the same stoichiometry. This heterogeneity between front surface and back surface does not originate from differences of accessibility to the reagent, but from the shape of electric current lines. The electric current at work electrode is bigger on the side that faces counter and reference electrodes, than on the other side (the "hidden" side).
Since the grafting process only occurs where electric current is, we observe higher front surface/back surface heterogeneity for thicker electrodes. This effect also causes some difficulties in controlling local potentials. Some experiments performed with much thicker films (buckypapers being 15 to $50 \mu \mathrm{m}$ thick)
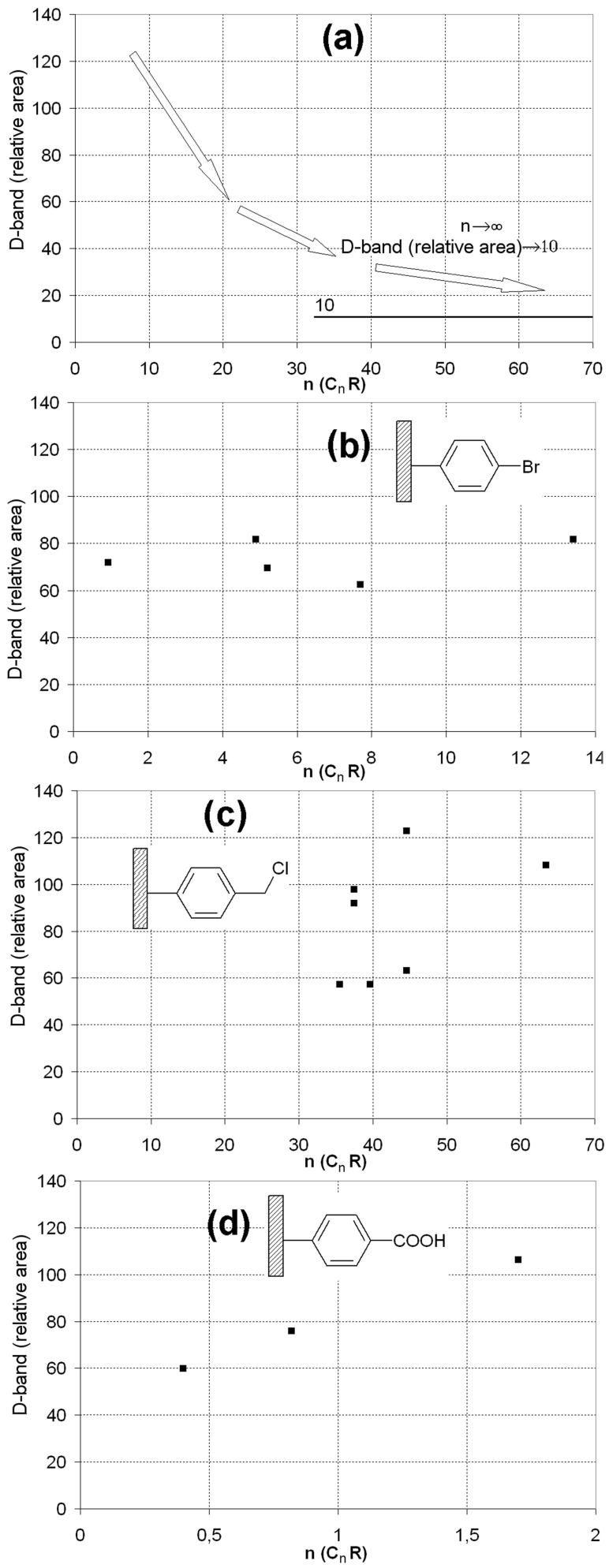

Fig. 5 Relative area of the D-band (Raman diffusion at $676 \mathrm{~nm}$ ) plotted against the stoichiometry derived from XPS measurements. Concerning D-bands, the area of the strongest tangential mode, at $1592 \mathrm{~cm}^{-1}$, is taken as a reference 100 . Figure 5a schematises the expected downward trend.

Figures $5 \mathrm{~b}, 5 \mathrm{c}$ and $5 \mathrm{~d}$ deal with the experiments performed with diazonium salts 1,3 and 4 respectively. 
yielded heterogeneously grafted zones.

The Figure $4 \mathrm{~b}$ shows the XPS spectrum of a film functionalised with 4-bromophenyl groups, whereas the Figure $4 \mathrm{a}$ is the spectrum of pristine nanotubes. The two upper inserts of this Figure plot the two peaks that were considered for the determination of the stoichiometry: $\mathrm{C} 1 \mathrm{~s}$ and $\mathrm{Br} 3 \mathrm{~d}$. The molar percentages obtained from the integration of these peaks are $\mathrm{C} 1 \mathrm{~s}$ $=92.0 \%$ and $\mathrm{Br} 3 \mathrm{~d}=8.0 \%$. Among these 92 carbon atoms, about 1 stems from amorphous carbon (as impurity of HipCo tubes) and $8 \times 6$ correspond to the grafted aryls. The number $\mathrm{n}$ is therefore 5.4: $C_{5.4}$ R. Different attempts performed with 4bromophenyl diazonium 1 led to stoichiometries ranging from $\mathrm{C}_{1} \mathrm{R}$ to $\mathrm{C}_{13} \mathrm{R}$ (depending on the thickness of the film). It must be emphasized that the previously reported experiments, using the same diazonium salt and the same nanotubes, yielded a $\mathrm{C}_{25} \mathrm{R}$ stoichiometry. ${ }^{26}$ Experiments performed with 2 yielded sulfur amounts that were hardly detectable with XPS (and the Raman spectra were unchanged after functionalisation). The stoichiometries were about $\mathrm{C}_{1000} \mathrm{R}$. These low ratio of grafted aryl groups on the nanotubes come essentially from the low solubility of the diazonium $\mathbf{2}$ in acetonitrile. Experiments with 3 led to stoichiometries ranging from $\mathrm{C}_{35} \mathrm{R}$ to $\mathrm{C}_{63} \mathrm{R}$, and from $\mathrm{C}_{0.4} \mathrm{R}$ to $\mathrm{C}_{1.6} \mathrm{R}$ in the case of the 4 compound. The values of $n$ concerning the diazonium compounds 1 and 4 (around 5, even below 2) are hardly explainable if we just consider a single layer of bonded aryl moieties. Some previous works about the fluorination of SWNTs ${ }^{34,35}$ showed that the lower limit value of $\mathrm{n}$ is 2: below that value, the tubular structure is altered contrarily to what we found from the TEM experiments. Moreover in our situation, grafted groups will cause higher steric hindering, the

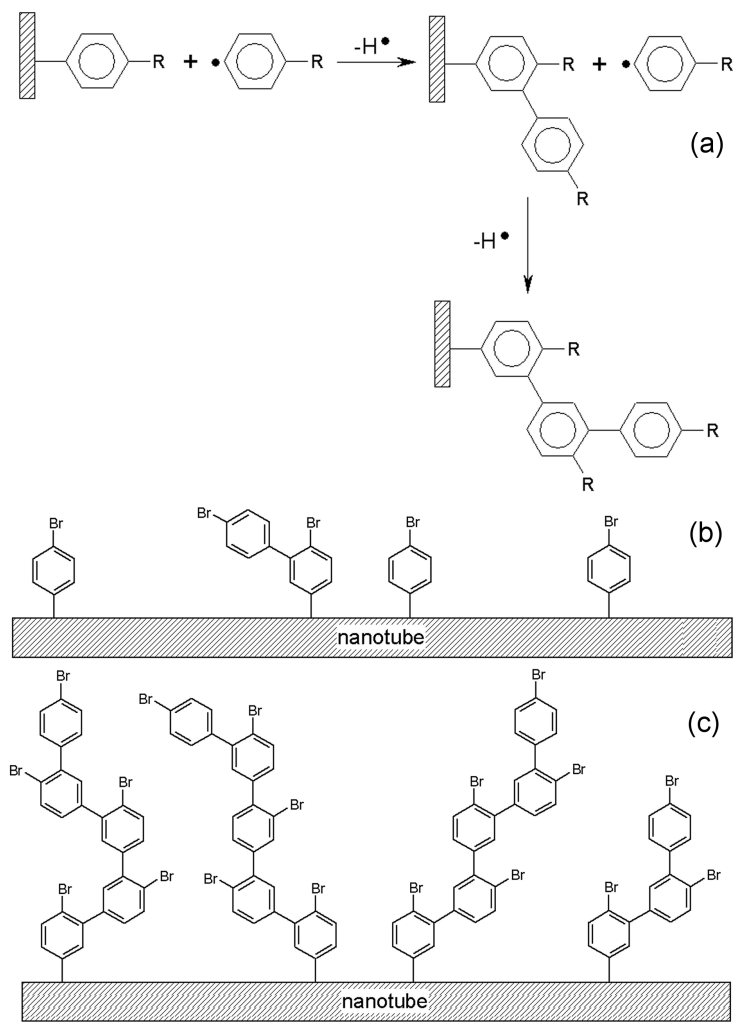

Fig. 6 (a) Grafting process of aryl radicals, in solution, onto aryl groups bound to a graphite surface. ${ }^{36}$ The hydrogen atoms produced by this mechanism may react either with solvent molecules, or with other aryl radicals, or with other hydrogen atoms. Illustrations (b) and (c) schematise two samples giving rise to D-bands that have the same intensity (same number of covalent bonds on the sidewalls of nanotubes), but to different bromine amounts in the XPS spectra. This difference can be explained through the growth of aryl chains on SWNTs. result being a lower limit for $\mathrm{n}$ above 2. Based on simple geometric consideration, we may expect values around 10 (in order to leave enough space between two adjacent grafted aryl groups). Such a difference cannot be explained by the presence of contaminant molecules (solvent, carbonates and hydrocarbons) adsorbed on the surface of the nanotubes film: if carbon atoms coming from adsorbed impurities were considered, the number $\mathrm{n}$ would be even lower. Furthermore, no signals attributed to nitrogen or chlorine, elements due to the solvents acetonitrile and chloroform, could be seen (Figure $4 \mathrm{~b}$ ). In order to understand the causes of theses differences, we compared the XPS and Micro-Raman data originated from the same sample. Figure 5 represents the plots of D-band integrated intensity against the stoichiometry number $\mathrm{n}$ derived from XPS for the different investigated compounds. The relative area of the Dband is connected with the number of covalent bonds created on the sidewalls, whereas $\mathrm{n}$ stems from the number of bromine bound to nanotubes. We expect that the relative area of the Dband that is proportional to the number of defaults on the sidewalls, decreases as the number $\mathrm{n}$ rises (Figure 5a). When $\mathrm{n}$ tends to the infinite (pristine nanotubes), the relative area of the D-band tends to 10 (measured value for pristine HipCo tubes). The data (Figures $5 \mathrm{~b}, \mathrm{c}, \mathrm{d}$ ) do not follow the expected downward trend. As an example, let us focus on the results of Figure $5 \mathrm{~b}$ : for different, nearly identical D-band values (areas around 70), we observe very different $\mathrm{n}$ values (ranging from 0.9 to 13 , depending on the film of nanotubes). Figure $5 \mathrm{~b}$ shows that large and different numbers of aryl groups can be attached on the nanotube without being seen by the surface itself and thus indicate that these "extra" aryl groups are not directly linked to the walls. These results highlight a polymerization phenomenon resulting in a structure where the aryl moieties have reacted on the first attached groups. At this point, we should remember that the electrochemical reduction of aryldiazonium salts produced highly reactive aryl radicals near the electrode (that is the principle of the method) that are able to react not only on the nanotube surface but also on any other aromatic rings such as the already bonded aryl moieties. Such a likely reaction, between the generated aryl radicals and the moieties bound to the carbon surface, has already been reported by Kariuki et al. in the case of a HOPG graphite surface (Figure 6a). ${ }^{36}$ As far as we are concerned, this process may explain the diagrams of the Figure 5 , as exemplified on the Figures $6 b$ and $c$, where the same number of nucleation sites on the sidewalls of SWNTs (same Dband values) would correspond to aryl chains of different lengths (different $n$ values).

This polymerization phenomenon was considered by Bahr et al., in their work about the electrochemical functionalisation of buckypapers, ${ }^{26}$ but was rejected by the authors because of steric hindering. This argument may eventually be justified for bulky grafting aryls, such as 4-tertbutylphenyl, but seems more questionable for aryl moieties such as 4-bromophenyl or 4carboxyphenyl. ${ }^{36}$ In the present experiments, our results provide new and clear evidences for the growth of aryl chains and the formation of multilayers of aryl moieties on the SWNTs for all the investigated diazonium salts.

\section{Conclusion}

The covalent bonding and growth of aryl chains on electrochemically functionalised nanotubes has been monitored by comparison of complementary XPS and Micro-Raman data. Raman diffusion gives information about the carbon backbone of SWNTs, whereas XPS informs about the quantity of grafted moieties. Such a polymerization phenomenon (growth of aryl chains) has already been described in literature, in the case of HOPG graphite. It is therefore not surprising to observe that similar process occurs in the case of carbon nanotubes. Highly reactive aryl radicals react first with certain sites of the sidewalls. Then these sites of initial attack and functionalisation 
act as nucleation sites for the subsequent growth of aryl chains. Some further studies will have to be done in order to control (or limit) the polymerization phenomenon, by tuning experimental parameters, such as the time during which the potential is applied or the volume (steric hindrance) of the grafted moieties. In spite of this phenomenon, the electrochemical functionalisation based on the electrogeneration of reactive radicals in the vicinity of the nanotube surface shows several advantages, compared to some other grafting methods, as the clean and non destructive chemical functionalisation or selective electrochemical modifications of individual objects. An example has been given by Knez et al: ${ }^{37}$ among different bundles of SWNTs deposited on a $\mathrm{Si} / \mathrm{SiO}_{2}$ substrate, only the bundles connected with an $\mathrm{Au} / \mathrm{Pd}$ electrode were modified. The electrochemical generation of radicals also gives the hope to make some selective chemistry on SWNTs, according to their electrical properties (metallic or semiconductive). In contrast, the electrochemical functionalisation has some limitations due to the fact that the reactions have to be performed on a thin film (about $2 \mu \mathrm{m}$ ), in order to get a homogeneous grafting throughout the nanotubes layer. Hence, this method is not well adapted to the functionalisation of large quantities of nanotubes. Furthermore, if the reaction is homogeneous at the scale of the XPS or MicroRaman probes, it may be heterogeneous at the scale of nanotubes bundles. Due to differences in the accessibility for the diazonium salt, SWNTs on the outer shell of a bundle are likely to be more grafted than nanotubes inside the bundle. The in-situ generation of the diazonium species seems an interesting alternative to the electrochemical method, ${ }^{38}$ since the grafting can be performed on dispersed nanotubes in solution. Diazonium species are generated from the aniline derivative and isoamyl nitrite. Larger amounts of SWNTs can therefore be functionalised and the problem of homogeneity is less critical.

\section{Acknowledgement}

Financial support from the CNRS and the Région Pays de Loire ( $\mathrm{PhD}$ fellowship for P. R. M.) is gratefully acknowledged. We thank V. Fernandez for assistance with the XPS measurements, C. Marhic for help with the TEM observations, J.-Y. Mévellec for participation in the Raman measurements and A. Barreau for help with the SEM observations.

\section{References}

1 P. M. Ajayan, Chem. Rev., 1999, 99, 1787-1799.

2 R. Saito, G. Dresselhaus and M. S. Dresselhaus, Physical Properties of Carbon Nanotubes, World Scientific, Singapore, 1998.

3 P. J. F. Harris, Carbon Nanotubes and Related Structures: New Materials for the Twenty-First Century, Cambridge University Press, Cambridge, 1999.

4 S. S. Wong, A. T. Woolley, E. Joselevich, C. L. Cheung, C. M. Lieber, J. Am. Chem. Soc., 1998, 120, 8557-8558.

5 J. Chen, A. M. Rao, S. Lyuksyutov, M. E. Itkis, M. A. Hamon, H. Hu, R. W. Cohn, P. C. Eklund, D. T. Colbert, R. E. Smalley, R. C. Haddon, J. Phys. Chem. B, 2001, 105, 2525-2528.

6 E. T. Mickelson, I. W. Chiang, J. L. Zimmerman, P. J. Boul, J. Lozano, J. Liu, R. E. Smalley, R. H. Hauge, J. L. Margrave, J. Phys. Chem. B, 1999, 103, 4318-4322.

7 J. Kong, M. G. Chapline, H. Dai, Adv. Mater., 2001, 13, 1384-1386.

8 Z. Liu, Z. Shen, T. Zhu, S. Hou, L. Ying, Langmuir, 2000, 16, 3569 3573.

9 D. Chattopadhyay, I. Galeska, F. Papadimitrakopoulos, J. Am. Chem. Soc., 2001, 123, 9451-9452.

10 S. Delpeux, K. Metenier, R. Benoit, F. Vivet, L. Boufendi, S. Bonnamy, F. Betgu, AIP Conference Proceedings, 1999, 486, 470473.

11 A. Hirsch, Angew. Chem. Int. Ed., 2002, 41, 1853-1859.

12 M. A. Hamon, J. Chen, H. Hu, Y. Chen, M. E. Itkis, A. M. Rao, P. C. Eklund, R. C. Haddon, Adv. Mater., 1999, 11, 834-840.

13 M. A. Hamon, H. Hu, P. Bhowmik, S. Niyogi, B. Zhao, M. E. Itkis, R. C. Haddon, Chem. Phys. Lett., 2001, 347, 8-12.
14 P. J. Boul, E. T. Mickelson, C. B. Huffman, L. M. Ericson, I. W. Chiang, K. A. Smith, D. T. Colbert, R. H. Hauge, J. L. Margrave, R. E. Smalley, Chem. Phys. Lett., 1999, 310, 367-372.

15 M. Holzinger, O. Vostrowsky, A. Hirsch, F. Hennrich, M. Kappes, R. Weiss, F. Jellen, Angew. Chem. Int. Ed., 2000, 40, 4002-4005.

16 X. Wang, Y. Liu, W. Qiu, D. Zhu, J. Mater. Chem., 2002, 12, 16361639.

17 B. Vigolo, A. Pénicaud, C. Coulon, C. Sauder, R. Pailler, C. Journet, P. Bernier, P. Poulin, Science, 2000, 290, 1331-1334.

18 M. J. O'Connell, P. J. Boul, L .M. Ericson, C. Huffman, Y. Wang, E. Haroz, C. Kuper, J. Tour, K. D. Ausman, R. E. Smalley, Chem. Phys. Lett., 2001, 342, 265-271.

19 B. W. Smith, M. Monthioux, D. E. Luzzi, Chem. Phys. Lett., 1999, 315, 31-36.

20 M. Delamar, R. Hitmi, J. Pinson, J.-M. Savéant, J. Am. Chem. Soc., 1992, 114, 5883-5884.

21 C. P. Andrieux, F. Gonzales, J. M. Savéant, J. Am. Chem. Soc., 1997, 119, 4292.

22 Y.-C. Liu, R. L. McCreery, J. Am. Chem. Soc., 1995, 117, $11254-$ 11259.

23 M. Delamar, G. Désarmot, O. Fagebaume, R. Hitmi, J. Pinson, J.-M. Savéant, Carbon, 1997, 35, 801-807.

24 E. Coulon, J. Pinson, J.-D. Bourzat, A. Commerçon, J. P. Pulicani, Langmuir, 2001, 17, 7102-7106.

25 J. A. Belmont, R. M. Amici, P. Galloway, Patent PCT Int. Appl. WO 9618688 Al, Cabot Corp.

26 J. L. Bahr, J. Yang, D. V. Kosynkin, M. J. Bronikowski, R. E. Smalley, J. M. Tour, J. Am. Chem. Soc., 2001, 123, 6536-6542.

27 S. E. Kooi, U. Schlecht, M. Burghard, K. Kern, Angew. Chem. Int. Ed., 2002, 41, 1353-1355.

28 P. Nikolaev, M. J. Bronikowski, R. K. Bradley, F. Rohmund, D. T. Colbert, K. A. Smith, R. E. Smalley, Chem. Phys. Lett., 1999, 313, 91-97.

29 E. Coulon, J. Pinson, J.-D. Bourzat, A. Commerçon, J. P. Pulicani, Langmuir, 2001, 17, 7102-7106.

30 P. R. Marcoux, J. Schreiber, P. Batail, S. Lefrant, J. Renouard, G. Jacob, D. Albertini, J.-Y. Mevellec, Phys. Chem. Chem. Phys., 2002, 4, 2278-2285.

31 John C. Vickerman, Surface Analysis: The Principal Techniques, John Wiley \& Sons Ltd, Chichester, 1997.

32 P. Cadman, G. Gossedge, J. D. Scott, J. Elect. Spectrosc. Rel. Phenom., 1978, 13, 1-6.

33 J. L. Bahr, E. T. Mickelson, M. J. Bronikowski, R. E. Smalley, J. M. Tour, Chem. Commun., 2001, 193-194.

34 E. T. Mickelson, C. B. Huffman, A. G. Rinzler, R. E. Smalley, R. H. Hauge, J. L. Margrave, Chem. Phys. Lett., 1998, 296, 188-194.

35 K. F. Kelly, I. W. Chiang, E. T. Mickelson, R. H. Hauge, J. L. Margrave, X. Wang, G. E. Scuseria, C. Radloff, N. J. Halas, Chem Phys. Lett., 1999, 313, 445-450.

36 J. K. Kariuki, M. T. McDermott, Langmuir, 1999, 15, 6534-6540.

37 M. Knez, M. Sumser, A. M. Bittner, C. Wege, H. Jeske, S. Kooi, M. Burghard, K. Kern, J. Electroanal. Chem., 2002, 522, 70-74.

38 J. L. Bahr, J. M. Tour, Chem. Mater., 2001, 13, 3823-3824. 
(') P. M. Ajayan, Chem. Rev., 1999, 99, 1787-1799.

$\left(^{2}\right)$ R. Saito, G. Dresselhaus and M. S. Dresselhaus, Physical Properties of Carbon Nanotubes, World Scientific, Singapore, 1998.

$\left.{ }^{3}\right)$ P. J. F. Harris, Carbon Nanotubes and Related Structures: New Materials for the Twenty-First Century, Cambridge University Press, Cambridge, 1999.

( $\left.{ }^{4}\right)$ S. S. Wong, A. T. Woolley, E. Joselevich, C. L. Cheung, C. M. Lieber, J. Am. Chem. Soc., 1998, 120, $8557-8558$.

$\left({ }^{5}\right)$ J. Chen, A. M. Rao, S. Lyuksyutov, M. E. Itkis, M. A. Hamon, H. Hu, R. W. Cohn, P. C. Eklund, D. T. Colbert, R. E. Smalley, R. C. Haddon, J. Phys. Chem. B, 2001, 105, 2525-2528.

$\left(^{6}\right)$ E. T. Mickelson, I. W. Chiang, J. L. Zimmerman, P. J. Boul, J. Lozano, J. Liu, R. E. Smalley, R. H. Hauge, J. L. Margrave, J. Phys. Chem. B, 1999, 103, 4318-4322.

$\left(^{7}\right)$ J. Kong, M. G. Chapline, H. Dai, Adv. Mater., 2001, 13, 1384-1386.

$\left.{ }^{8}\right)$ Z. Liu, Z. Shen, T. Zhu, S. Hou, L. Ying, Langmuir, 2000, 16, 3569-3573.

$\left({ }^{9}\right)$ D. Chattopadhyay, I. Galeska, F. Papadimitrakopoulos, J. Am. Chem. Soc., 2001, 123, 94519452.

$\left({ }^{10}\right)$ S. Delpeux, K. Metenier, R. Benoit, F. Vivet, L. Boufendi, S. Bonnamy, F. Betgu, AIP Conference Proceedings, 1999, 486, 470-473.

( $\left.{ }^{11}\right)$ A. Hirsch, Angew. Chem. Int. Ed., 2002, 41, 1853-1859.

$\left.{ }^{12}\right)$ M. A. Hamon, J. Chen, H. Hu, Y. Chen, M. E. Itkis, A. M. Rao, P. C. Eklund, R. C. Haddon, Adv. Mater., 1999, 11, 834-840. 
$\left({ }^{13}\right)$ M. A. Hamon, H. Hu, P. Bhowmik, S. Niyogi, B. Zhao, M. E. Itkis, R. C. Haddon, Chem. Phys. Lett., 2001, 347, 8-12.

$\left({ }^{14}\right)$ P. J. Boul, E. T. Mickelson, C. B. Huffman, L. M. Ericson, I. W. Chiang, K. A. Smith, D. T. Colbert, R. H. Hauge, J. L. Margrave, R. E. Smalley, Chem. Phys. Lett., 1999, 310, 367-372.

$\left({ }^{15}\right)$ M. Holzinger, O. Vostrowsky, A. Hirsch, F. Hennrich, M. Kappes, R. Weiss, F. Jellen, Angew. Chem. Int. Ed., 2000, 40, 4002-4005.

(16) X. Wang, Y. Liu, W. Qiu, D. Zhu, J. Mater. Chem., 2002, 12, 1636-1639.

$\left({ }^{17}\right)$ B. Vigolo, A. Pénicaud, C. Coulon, C. Sauder, R. Pailler, C. Journet, P. Bernier, P. Poulin, Science, 2000, 290, 1331-1334.

$\left({ }^{18}\right)$ M. J. O’Connell, P. J. Boul, L .M. Ericson, C. Huffman, Y. Wang, E. Haroz, C. Kuper, J. Tour, K. D. Ausman, R. E. Smalley, Chem. Phys. Lett., 2001, 342, 265-271.

(19) B. W. Smith, M. Monthioux, D. E. Luzzi, Chem. Phys. Lett., 1999, 315, 31-36.

$\left({ }^{20}\right)$ M. Delamar, R. Hitmi, J. Pinson, J.-M. Savéant, J. Am. Chem. Soc., 1992, 114, 5883-5884.

$\left({ }^{21}\right)$ P. Allongue, M. Delamar, B. Desbat, O. Fagebaume, R. Hitmi, J. Pinson, J. M. Savéant, J. Am. Chem. Soc., 1997, 119, 201.

(22) Y.-C. Liu, R. L. McCreery, J. Am. Chem. Soc., 1995, 117, 11254-11259.

(23) M. Delamar, G. Désarmot, O. Fagebaume, R. Hitmi, J. Pinson, J.-M. Savéant, Carbon, 1997, 35, 801-807.

$\left({ }^{24}\right)$ E. Coulon, J. Pinson, J.-D. Bourzat, A. Commerçon, J. P. Pulicani, Langmuir, 2001, 17, 71027106.

(25) J. A. Belmont, R. M. Amici, P. Galloway, Patent PCT Int. Appl. WO 9618688 A1, Cabot Corp. 
$\left({ }^{26}\right)$ J. L. Bahr, J. Yang, D. V. Kosynkin, M. J. Bronikowski, R. E. Smalley, J. M. Tour, J. Am. Chem. Soc., 2001, 123, 6536-6542.

${ }^{(27)}$ S. E. Kooi, U. Schlecht, M. Burghard, K. Kern, Angew. Chem. Int. Ed., 2002, 41, 1353-1355.

$\left.{ }^{28}\right)$ P. Nikolaev, M. J. Bronikowski, R. K. Bradley, F. Rohmund, D. T. Colbert, K. A. Smith, R. E. Smalley, Chem. Phys. Lett., 1999, 313, 91-97.

$\left({ }^{29}\right)$ E. Coulon, J. Pinson, J.-D. Bourzat, A. Commerçon, J. P. Pulicani, Langmuir, 2001, 17, 71027106.

$\left({ }^{30}\right)$ P. R. Marcoux, J. Schreiber, P. Batail, S. Lefrant, J. Renouard, G. Jacob, D. Albertini, J.-Y. Mevellec, Phys. Chem. Chem. Phys., 2002, 4, 2278-2285.

${ }^{(31)}$ John C. Vickerman, Surface Analysis: The Principal Techniques, John Wiley \& Sons Ltd, Chichester, 1997.

(32) P. Cadman, G. Gossedge, J. D. Scott, J. Elect. Spectrosc. Rel. Phenom., 1978, 13, 1-6.

(33) J. L. Bahr, E. T. Mickelson, M. J. Bronikowski, R. E. Smalley, J. M. Tour, Chem. Commun., 2001, 193-194.

$\left({ }^{34}\right)$ E. T. Mickelson, C. B. Huffman, A. G. Rinzler, R. E. Smalley, R. H. Hauge, J. L. Margrave, Chem. Phys. Lett., 1998, 296, 188-194.

$\left({ }^{35}\right)$ K. F. Kelly, I. W. Chiang, E. T. Mickelson, R. H. Hauge, J. L. Margrave, X. Wang, G. E. Scuseria, C. Radloff, N. J. Halas, Chem. Phys. Lett., 1999, 313, 445-450.

$\left.{ }^{(36}\right)$ J. K. Kariuki, M. T. McDermott, Langmuir, 1999, 15, 6534-6540.

$\left({ }^{37}\right)$ M. Knez, M. Sumser, A. M. Bittner, C. Wege, H. Jeske, S. Kooi, M. Burghard, K. Kern, J. Electroanal. Chem., 2002, 522, 70-74. 
$\left({ }^{38}\right)$ J. L. Bahr, J. M. Tour, Chem. Mater., 2001, 13, 3823-3824. 